\title{
Aeromonas jandaei
}

National Cancer Institute

\section{Source}

National Cancer Institute. Aeromonas jandaei. NCI Thesaurus. Code C86126.

A species of facultatively anaerobic, Gram negative, rod shaped bacteria assigned to the phylum Proteobacteria. This bacteria is oxidase positive, does not hydrolyze esculin, produces gas from glucose and produces indole and mannitol. A. jandaei is an opportunistic pathogen found in soil and aquatic environments. 